Article

\title{
Formation Design for Single-Pass GEO InSAR Considering Earth Rotation Based on Coordinate Rotational Transformation
}

\author{
Zhiyang Chen ${ }^{1} \mathbb{0}$, Xichao Dong ${ }^{1,2,3}$, Yuanhao $\mathrm{Li}^{4, * \mathbb{C}}$ and Cheng $\mathrm{Hu}^{1,3}$ \\ 1 School of Information and Electronics, Beijing Institute of Technology, Beijing 100081, China; \\ 3120160358@bit.edu.cn (Z.C.); xcdong@bit.edu.cn (X.D.); cchchb@bit.edu.cn (C.H.) \\ 2 Key Laboratory of Electronic and Information Technology in Satellite Navigation (Beijing Institute of \\ Technology), Ministry of Education, Beijing 100081, China \\ 3 Beijing Institute of Technology Chongqing Innovation Center, Chongqing 401120, China \\ 4 Department of Geoscience and Remote Sensing, Delft University of Technology, 2628 Delft, The Netherlands \\ * Correspondence: Y.Li-24@tudelft.nl
}

Received: 17 December 2019; Accepted: 7 February 2020; Published: 8 February 2020

\begin{abstract}
The single-pass geosynchronous synthetic aperture radar interferometry (GEO InSAR) adopts the formation of a slave satellite accompanying the master satellite, which can reduce the temporal decorrelation caused by atmospheric disturbance and observation time gap between repeated tracks. Current formation design methods for spaceborne SAR are based on the Relative Motion Equation (RME) in the Earth-Centered-Inertial (ECI) coordinate system (referred to as ECI-RME). Since the Earth rotation is not taken into account, the methods will lead to a significant error for the baseline calculation while applied to formation design for GEO InSAR. In this paper, a formation design method for single-pass GEO InSAR based on Coordinate Rotational Transformation (CRT) is proposed. Through CRT, the RME in Earth-Centered-Earth-Fixed (ECEF) coordinate system (referred to as ECEF-RME) is derived. The ECEF-RME can be used to describe the accurate baseline of close-flying satellites for different orbital altitudes, but not limited to geosynchronous orbit. Aiming at the problem that ECEF-RME does not have a regular geometry as ECI-RME does, a numerical formation design method based on the minimum baseline error criterion is proposed. Then, an analytical formation design method is proposed for GEO InSAR, based on the Minimum Along-track Baseline Criterion (MABC) subject to a fixed root mean square of the perpendicular baseline. Simulation results verify the validity of the ECEF-RME and the analytical formation design method. The simulation results also show that the proposed method can help alleviate the atmospheric phase impacts and improve the retrieval accuracy of the digital elevation model (DEM) compared with the ECI-RME-based approach.

Keywords: GEO InSAR formation design; Earth rotation; Coordinate Rotational Transformation (CRT); Relative Motion Equation in ECEF coordinate system (ECEF-RME); Minimum Along-track Baseline Criterion (MABC)
\end{abstract}

\section{Introduction}

Geosynchronous synthetic aperture radar (GEO SAR) is a spaceborne SAR system operating at geosynchronous orbit with a height of about $36,000 \mathrm{~km}[1,2]$. Compared with the traditional low Earth orbit SAR (LEO SAR), GEO SAR has the advantages of a short revisit period (several hours to one day) and extensive coverage (imaging swath wider than $2000 \mathrm{~km}$ ). Additionally, the baseline brought by orbit perturbation can be adopted to realize the applications of SAR interferometry (InSAR) [3], SAR tomography (TomoSAR), etc. [4], so it has significant application potential for disaster prevention and mitigation, including flood disaster, geological disaster, etc. [1,5-7]. 
The performance of InSAR and TomoSAR based on monostatic GEO SAR significantly degrades due to temporal decorrelation (caused by the scene scattering fluctuation during the observation time gap between repeated tracks) and atmospheric disturbance. Similar to LEO SAR [8], we can use the formation GEO SAR to form a real-time baseline to improve interferometry performance $[9,10]$, realizing single-pass GEO InSAR. Additionally, multiple phase centers can be generated in GEO SAR formation, and a flexible baseline configuration can enrich the functions.

The primary task of SAR formation is to design the satellite orbital elements to obtain a reasonable formation configuration and then meet the application performance requirements. The formation design of LEO SAR has been adequately studied, and a very mature solution has been found. A very typical method is to describe the geometry of the formation using the Relative Motion Equation (RME) based on the difference between the master satellite's and slave satellite's orbital elements, and combining the mission requirements to design the satellite's orbital elements [11].

The theoretical study of the Relative Motion Equation has gone through three stages. The earliest form of RME, which was used to complete spacecraft rendezvous and docking tasks [12], was obtained by solving the Hill equation in the 1960s. However, the solving process is complicated, and the analytical solution has a relatively limited scope of application. To solve this problem, in 2002, Hanspeter Schaub of Orion International Technologies in the United States used the orbital element differences between master and slave satellites to describe the relative motion geometry between satellites [13]. The RME of this form can easily correspond to many typical configurations. This result has been widely used in the research of SAR missions. Subsequently, Giancarmine Fasano of the University of Naples Federico II in Italy, as a representative of the scholars, made a breakthrough in the theoretical research of the RME affected by the perturbation. In 2006, he proposed an approximate RME considering the influence of the J2 perturbation, with an error of only $0.1 \%$ [14], which can be used to optimize the typical configurations further. In 2007, he proposed a second-order analytical RME, which can be applied to a much wider range of bistatic angle of the formation, thus can be used to design the configurations for bistatic observations $[15,16]$.

In the aspect of the application of RME for spaceborne SAR formation design, most scholars use it to propose various configurations or to evaluate system performances. In [17], the regular hexagon configuration was proposed. By using the RME, many typical InSAR formations are proposed, including Cartwheel [18], Pendulum, Carpe [19], and Helix configurations [8]. Most of the subsequent InSAR formation design studies in LEO SAR are only to refine these typical configurations. In 2012, with respect to TomoSAR application, Giancarmine Fasano used the RME to optimize the multiple Cartwheel/Pendulum/Helix configurations to realize uniform samplings in the height direction, and found that only slight adjustment of the right ascension of ascending node (RAAN) and eccentricity were needed [20]. In [21], the maximum detective velocity, minimum detective velocity, elevation sensitivity, and elevation ambiguity indexes were associated mathematically with formation geometry parameters, and a multiple-satellite formation for GMTI and InSAR was designed. In [22], taking the optimal baseline of InSAR as an index, a satellite formation with the best DEM measurement accuracy was designed by using the method based on mean elements. In [23], the perturbation effect of the J2 term of the Earth is considered, and the ant colony algorithm is used to optimize the formation of 3-satellite so that the baseline of formation is as stable as possible and the DEM measurement accuracy can be improved. In [24], formation baseline, available orbit duty for InSAR, and latitude coverage are considered, and the influence of orbital elements on Cartwheel configuration is analyzed, to evaluate the feasibility of adding formation satellites for Cosmo/Skymed. Reference [25] analyzed the InSAR baseline, ground coverage, and imaging resolution of typical configuration, to evaluate the feasibility of launching nanosatellites CANX-4 and CANX-5.

The above RME equation is defined in the Earth-Centered-Inertial (ECI) coordinate system (referred to as ECI-RME in this paper). Thus, the baseline is calculated according to the satellite velocity in the ECI coordinate system. However, GEO SAR is severely affected by the Earth rotation. Therefore, the baseline needs to be calculated based on the satellite velocity in the Earth-Centered-Earth-Fixed 
(ECEF) coordinate system. Using ECI-RME will bring significant errors to the baseline calculation, so the performances of the designed formation based on ECI-RME will also be far from the requirements. This issue will be addressed in detail in the following context. In [16], the optimal baseline of GEO SAR formation is designed by using the Hill equation, but the effect of Earth rotation has not been considered. There is no other literature on SAR formation design.

To solve the problem that the ECI-RME cannot be applied to GEO InSAR formation design directly, this paper proposes a formation design method of single-pass GEO InSAR based on Coordinate Rotational Transformation (CRT). By this method, the Relative Motion Equation in the ECF coordinate system (called ECEF-RME) is obtained. Firstly, it is proved that the ECEF-RME and ECI-RME satisfy the relationship of coordinate rotational transformation, and the ECEF-RME is derived after calculating the rotation transformation matrix. This equation can be used to describe the accurate baseline of close-flying satellites for different orbital altitudes, but not limited to geosynchronous orbit. However, different from ECI-RME, ECEF-RME does not show apparent geometric features. Aiming at this problem, a general numerical optimization method for formation design based on the minimum baseline error criterion is proposed. Then, in GEO SAR, the Minimum Along-track Baseline Criterion (MABC) subject to a fixed root mean square of the perpendicular baseline is proposed, through which an analytical method for GEO InSAR formation design is given. Finally, the simulation results verify the validity of the ECEF-RME and the analytical design method. The simulation also shows that the proposed method can help alleviate the atmospheric disturbance, improve the digital elevation model (DEM) retrieval accuracy compared with ECI-RME-based approach.

The structure of this paper is as follows. In Section 2, we describe the GEO InSAR formation and design criteria. In Section 3, we give an analysis of the influence of Earth rotation on baseline calculation and then derive the ECEF-RME based on CRT. In Section 4, we propose the GEO InSAR formation design method based on MABC. Simulation is conducted in Section 5 to verify the accuracy of ECEF-RME and the validity of the GEO InSAR formation design method, and the advantages of the proposed method in the InSAR application are also shown. The advantages are further discussed in Section 6. We conclude this paper in Section 7.

\section{GEO InSAR Formation and Design Criteria}

We consider the GEO InSAR formation shown in Figure 1a. The system consists of a master satellite and a slave satellite. The master satellite can both transmit and receive signals, while the slave satellite serves as a passive receiver. The nadir tracks of the formation are as shown in Figure $1 \mathrm{~b}$ (the inter-satellite distance is exaggerated. It is much smaller in practice). The maximum inter-satellite distance is several hundred kilometers, depending on the critical baseline for InSAR. The critical baseline refers to the maximum baseline to ensure that the correlation coefficient of the image pairs is just reduced to zero, which can be expressed as $B_{\mathrm{c}}=\lambda R B_{w} \tan \theta_{i} / c$ [26], where $\lambda$ is the wavelength, $R$ being the slant range, $\theta_{i}$ being the incidence angle, $B_{w}$ being the bandwidth of the transmitted signal, $c$ being the light speed. Taking as an example, the typical wavelength $24 \mathrm{~cm}$ in L band, a bandwidth of $18 \mathrm{MHz}$, an incidence angle of $30^{\circ}$, the critical baseline is around $303 \mathrm{~km}$.

For the atmospheric disturbance, L-band GEO SAR is severely affected by the ionosphere, which will cause 2D image migration, and $500 \mathrm{~s}$ of integration time can cause a certain degree of azimuth defocusing [27]. The advantage of InSAR using formation is that temporal decorrelation and atmospheric disturbance can be significantly alleviated. Besides, since the revisit time of GEO SAR is only $24 \mathrm{~h}$, a large number of InSAR data can generate interferogram sequences, which provide the possibility for deformation observation and retrieval of deformation velocity. 


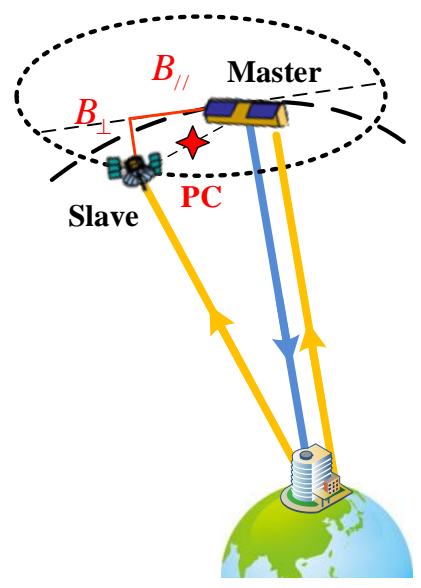

(a)

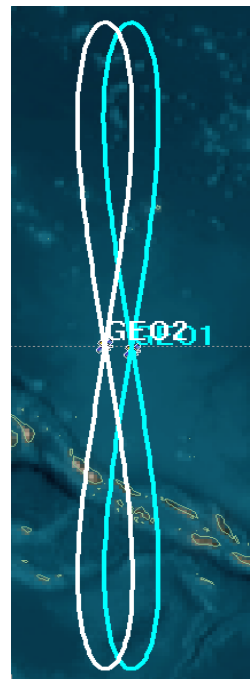

(b)

Figure 1. GEO InSAR formation. (a) Sketch map; (b) Nadir tracks.

Two of the essential factors in the formation design of GEO InSAR are to minimize the along-track baseline and to optimize the DEM retrieval accuracy. In interferometry, the main baseline components of formation include vertical baseline and along-track baseline, which are the components perpendicular to the slant range plane and the motion direction, respectively. The vertical baseline is necessary for DEM retrieval. In contrast, the along-track baseline brings a line of sight (LOS) difference between satellites in the azimuth direction, thus introduces rotation decorrelation, which should be eliminated as much as possible $[3,26]$. InSAR process is a maximum likelihood estimation, and the most important performance is the accuracy of DEM retrieval, which is related to the correlation coefficient $\gamma$ of the image pair, and the size of the vertical baseline $B_{\perp}$. The Cramer-Rao Lower Bound (CRLB) of the DEM retrieval accuracy can be expressed as $\sigma_{h}=\lambda R \sqrt{1-\gamma^{2}} /\left(4 \sqrt{2 L} \pi B_{\perp} \gamma\right)$, where $L$ is the number of looks. Furthermore, without considering the temporal decorrelation and the atmospheric disturbance, the correlation coefficient is related to the signal-to-noise ratio (SNR) $R_{S N}$ of the image, and the vertical baseline. Therefore, there is a vertical baseline that achieves optimal DEM retrieval accuracy. This baseline is called the "optimal baseline," which can be expressed as [28]:

$$
B_{\perp, o p t}=\left[1-\left(0.618-1.171 R_{S N}^{-1}\right)\left(1+R_{S N}^{-1}\right)\right] \frac{2 \lambda R B_{w} \tan \theta_{i}}{c}
$$

Therefore, to optimize the performances of GEO InSAR formation, we need to reduce the along-track baseline as much as possible, while the vertical baseline should be as close to the optimal baseline as possible. The master satellite of the formation is selected with an inclination of $16^{\circ}$ [29], which has an excellent coverage performance of low and medium latitude regions (such as China), so we only need to design the slave satellite's orbital elements.

\section{Influence of Earth Rotation and ECEF-RME Derivation}

\subsection{Influence of Earth Rotation on Baseline Calculation}

\subsubsection{ECI-RME and Corresponding Formation Design Method}

In satellite formations, ECI-RME (or the Hill equation) is usually used to describe the relative motion between satellites $[30,31]$. This equation describes the position of the slave satellite relative to the master satellite w.r.t. time. In ECI-RME, the origin is located in the master satellite, and the position of the slave satellite relative to the master satellite is represented by three orthogonal components in $X$, $\mathrm{Y}$, and $\mathrm{Z}$ directions. The coordinate system composed of $\mathrm{X}-\mathrm{Y}-\mathrm{Z}$ three directions is called the spacecraft 
orbit coordinate system (OCS) (referred to as ECI-OCS, since it is defined in the ECI coordinate system), also called the Hill frame [31], as shown in Figure 2. The X-axis is along the radial direction from the Earth center to the master satellite. The X-Y plane is the orbit motion plane, and the Y-axis points to the velocity side of the master satellite. If the eccentricity is zero, then the Y-axis has the same direction as the velocity. The Z-axis is perpendicular to the orbit motion plane, and $\mathrm{X}-\mathrm{Y}-\mathrm{Z}$ is a right-handed coordinate system.

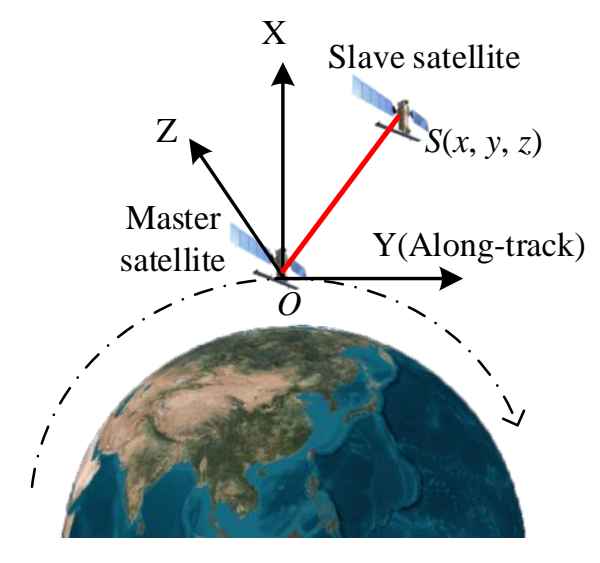

Figure 2. Spacecraft orbit coordinate system.

The three components of the ECI-RME can be expressed as:

$$
\left\{\begin{array}{l}
x(t)=-A_{1} \cos \left(n \cdot t+\alpha_{1}\right) \\
y(t)=2 A_{1} \sin \left(n \cdot t+\alpha_{1}\right)+l \\
z(t)=A_{2} \cos \left(n \cdot t+\alpha_{2}\right)
\end{array}\right.
$$

where $n$ is the angular velocity of the master satellite. $A_{1}, \alpha_{1}, l, A_{2}, \alpha_{2}$ are related to the orbital elements of the master and slave satellites [30], which satisfy:

$$
\begin{aligned}
& A_{1}=a \sqrt{\left(e_{d} \cos \Delta M-e_{c}\right)^{2}+\left(e_{d} \sin \Delta M\right)^{2}} \\
& A_{2}=-a \sqrt{\left(\Delta \Omega \sin i_{c}\right)^{2}+(\Delta i)^{2}} \\
& l=a\left[\Delta \omega-\Delta M+\Delta \Omega \cos i_{c}\right] \\
& \sin \left(\alpha_{1}-M_{c}\right)=e_{d} \sin \Delta M \cdot a / A_{1} \\
& \cos \left(\alpha_{1}-M_{c}\right)=\left(e_{d} \cos \Delta M-e_{c}\right) \cdot a / A_{1} \\
& \sin \left(\alpha_{2}-M_{c}-\omega_{c}\right)=\Delta i \cdot a / A_{2} \\
& \cos \left(\alpha_{2}-M_{c}-\omega_{c}\right)=\Delta \Omega \sin i_{c} \cdot a / A_{2}
\end{aligned}
$$

where $a, e, i, \Omega, \omega$ and $M$ are semi-major axis, eccentricity, inclination, right ascension of the ascending node (RAAN), the argument of perigee (AOP), and the mean anomaly of one satellite. These variables are the six orbital elements determining the motion of a spacecraft. The subscript $c$ and $d$ represent the center spacecraft (master satellite in the paper) and the slave satellite, respectively. $\Delta \sigma=\sigma_{d}-\sigma_{c}$ is the orbital element difference of the master and slave satellite, where $\sigma$ can be an arbitrary element mentioned above. Generally, the master and slave satellites have the same semi-major axis so that the formation is stable.

The along-track and perpendicular baseline can be expressed as:

$$
\begin{aligned}
& B_{A T}(t)=|y(t)| \\
& B_{\perp}(t)=\left|z(t) \cos \theta_{d}-x(t) \sin \theta_{d}\right|
\end{aligned}
$$

where $\theta_{d}$ represents the off-nadir angle. 
Equation (1) shows that the three components of X-Y-Z vary periodically with the satellite motion time, and the variation period is consistent with the orbital period. It can be seen that the ECI-RME has a very apparent geometric meaning. In the orbit plane (i.e., $X-Y$ plane), the slave satellite moves in an elliptical motion w.r.t. the master satellite. The center of the ellipse is in the along-track direction of the master satellite, with a distance of $l$. The ratio of the major axis and the minor axis of the ellipse is 2:1. The slave satellite moves in a simple harmonic motion in the direction perpendicular to the orbit plane, i.e., $Z$ direction. The ECI-RME can be characterized by five geometry parameters, including the minor semi-axis $A_{1}$ of the elliptical motion, the initial phase $\alpha_{1}$ of the elliptical motion, the along-track distance $l$ of the elliptical motion trajectory center, the amplitude $A_{2}$ of the harmonic motion, and the initial phase $\alpha_{1}-\alpha_{2}$ of the harmonic motion [32].

Usually, the orbital elements of the master satellite are determined, and the objective of the formation design is to determine the orbital elements of the salve satellite according to the application requirement. In the current literature, satellite formations are primarily designed based on the geometry parameters of ECI-RME or the expression of the baseline. In the geometry parameter-based design method, the Cartwheel configuration and the Pendulum configuration can be obtained by setting the amplitude of the harmonic motion, $A_{2}$, and the minor semi-axis of the elliptical motion, $A_{1}$, to be zero, respectively [18,24]. In the baseline expression-based design method, since the baseline varies over the entire orbit, the existing studies mainly design the orbital elements of the salve satellite to meet the baseline requirement at certain orbit positions, such as the initial orbit position [22] or above the equator [20].

\subsubsection{Influence of Earth Rotation on Baseline Calculation}

In the ECI-RME, the y-axis has the same direction as the satellite velocity, that is, the direction of velocity in the ECI coordinate system, $\vec{v}_{\mathrm{ECI}}(t)$. However, the effective velocity of the SAR imaging satellite is the velocity of the satellite relative to the scene; that is, the velocity in the ECEF coordinate system, $\vec{v}_{\mathrm{ECEF}}(t)$. Therefore, the calculation of the along-track baseline and perpendicular baseline should be based on the direction of $\vec{v}_{\mathrm{ECEF}}(t)$. The projection of the baseline in $\vec{v}_{\mathrm{ECEF}}(t)$ direction is the along-track baseline, and the perpendicular baseline is determined by the baseline component perpendicular to $\vec{v}_{\mathrm{ECEF}}(t)$ and the off-nadir angle. There is a velocity difference between $\vec{v}_{\mathrm{ECI}}(t)$ and $\vec{v}_{\mathrm{ECEF}}(t)$ caused by the Earth rotation, as follows:

$$
\vec{v}_{\mathrm{ECEF}}(t)=\vec{v}_{\mathrm{ECI}}(t)-\vec{n}_{\mathrm{E}} \times \vec{r}(t)
$$

where $\vec{n}_{\mathrm{E}}$ is the angular velocity of the Earth, and $\vec{r}(t)$ is the vector from the center of the Earth to the satellite.

It is worth noting that the baseline calculation method based on $\vec{v}_{\mathrm{ECEF}}(t)$ is accurate for LEO SAR, Medium-Earth-Orbit (MEO) SAR, and GEO SAR. Furthermore, whether $\vec{v}_{\mathrm{ECI}}(t)$ can be used as an approximate replacement depends on whether the Earth rotation will bring a significant direction difference between $\vec{v}_{\mathrm{ECEF}}(t)$ and $\vec{v}_{\mathrm{ECI}}(t)$. In LEO SAR, because $\vec{r}(t)$ is relatively small, $\vec{v}_{\mathrm{ECEF}}(t)$ and $\vec{v}_{\mathrm{ECI}}(t)$ are very close in size and direction, that is to say, the influence of Earth rotation can be ignored, so it is accurate enough to use $\vec{v}_{\mathrm{ECI}}(t)$ to calculate the baseline and then design formation configuration. However, the semi-major axis of GEO SAR is increased by two orders of magnitude, $\vec{v}_{\text {ECEF }}(t)$ and $\vec{v}_{\mathrm{ECI}}(t)$ are quite different, especially in the direction, so the Earth rotation cannot be ignored.

The formula of the angle between $\vec{v}_{\mathrm{ECEF}}(t)$ and $\vec{v}_{\mathrm{ECI}}(t), \theta$, in the case of small eccentricity, will be derived in the next sub-section, with the result shown in (11). Here we present the result in LEO SAR and GEO SAR in advance in Figure 3 to show the impacts of the Erath's rotation on the baseline calculation. The orbital elements are shown in Table 1, where the orbital elements of LEO SAR are similar to that of TerraSAR-X. Supposing a formation without an along-track baseline (suitable for InSAR application) in the ECI coordinate system has the perpendicular baseline of $B_{\perp}$, the relative 
baseline error caused by the Earth's rotation is $\left(B_{\perp}-\cos \theta B_{\perp}\right) / B_{\perp}=1-\cos \theta$. For LEO SAR, the angle between the directions of $\vec{v}_{\mathrm{ECEF}}(t)$ and $\vec{v}_{\mathrm{ECI}}(t)$ is not more than $4^{\circ}$, whose corresponding relative baseline error is $0.24 \%$. However, in GEO SAR, the angle can reach $80^{\circ}$, whose corresponding relative baseline error is $82.6 \%$, which will lead to the conversion of the along-track baseline to the cross-track baseline and vice versa. Therefore, in GEO SAR, the baseline component cannot be calculated accurately with $\vec{v}_{\mathrm{ECI}}(t)$.

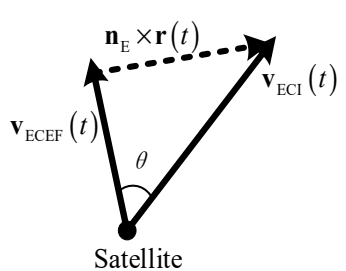

(a)

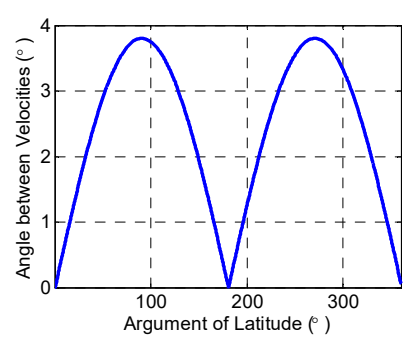

(b)

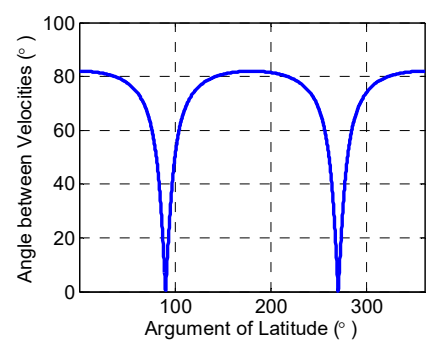

(c)

Figure 3. Angle between velocities in ECF and ECI coordinate. (a) Definition of the angle; (b) the angle in LEO SAR; (c) the angle in GEO SAR.

Table 1. Orbital elements for simulation.

\begin{tabular}{ccccccc}
\hline \multirow{2}{*}{ Orbital Element } & \multicolumn{2}{c}{ LEO SAR } & \multicolumn{2}{c}{ MEO SAR } & \multicolumn{2}{c}{ GEO SAR } \\
\cline { 2 - 6 } & Master & Slave & Master & Slave & Master & Slave \\
\hline Semi-major axis $(\mathrm{km})$ & 7003.52 & 7003.52 & 30,000 & 30,000 & 42,164 & 42,164 \\
Eccentricity & 0.00118 & 0.00118 & 0 & 0 & 0 & 0 \\
Inclination $\left(^{\circ}\right)$ & 97.86 & 97.86 & 112 & 112 & 16 & 16 \\
AOP $\left(^{\circ}\right)$ & 90 & 90 & 0 & 0 & 0 & 0 \\
RAAN $\left(^{\circ}\right)$ & 0 & 0.01 & 0 & 0.05 & 0 & 0.25 \\
Mean anomaly $\left(^{\circ}\right)$ & 0 & 0 & 0 & 0 & 0 & 0.1 \\
\hline
\end{tabular}

\subsection{Derivation of ECEF-RME Based on CRT}

It can be seen from the previous section that the Earth rotation results in a significant error in calculating the baseline of GEO SAR using the ECI-RME. To correctly calculate the baseline component under the influence of Earth rotation, we need to derive the Relative Motion Equation under the ECEF coordinate system. For this purpose, we firstly establish a coordinate system with $\vec{v}_{\mathrm{ECEF}}(t)$ as the coordinate axis, which we refer to as the orbital coordinate system in the ECEF coordinate system (ECEF-OSC). The orbital coordinate system with $\vec{v}_{\mathrm{ECI}}(t)$ as an axis is ECI-OSC, which has illustrated in Section 3.1. In ECEF-OSC $O-X^{\prime} Y^{\prime} Z^{\prime}$, the $Y^{\prime}$-axis coincides with $\vec{v}_{\mathrm{ECEF}}(t)$ in the positive direction, and the component of the spatial baseline in the $Y^{\prime}$-axis is the along-track baseline after considering the Earth rotation.

Because ECEF-OSC and ECI-OSC have the same origin, the relationship between the two coordinate systems can be expressed by the rotation transformation. By calculating the rotation matrix, the representation of spatial baseline in $O-X^{\prime} Y^{\prime} Z^{\prime}$ coordinate system can be obtained. It is worth noting that $\vec{v}_{\mathrm{ECEF}}(t)$ is still in the Y-Z plane of ECI-OSC, that is to say, in the two coordinate systems, $X^{\prime}$ and $X$ are coincident. We can calculate the inner product of $\vec{v}_{\mathrm{ECEF}}(t)$ and $X$-axis to verify this conclusion. Let $\hat{x}, \hat{y}$ and $\hat{z}$ represent the unit vectors of $X, Y$, and $Z$ axes, respectively. Then it is easy to check that $\left\langle\vec{v}_{\mathrm{ECEF}}(t), \hat{x}\right\rangle=\left\langle\vec{v}_{\mathrm{ECI}}(t) \cdot \hat{y}-\vec{n}_{\mathrm{E}} \times \hat{x} \cdot r(t), \hat{x}\right\rangle=0$. An inner product of 0 indicates that $\vec{v}_{\mathrm{ECEF}}(t)$ has no component in the $\mathrm{x}$-axis direction, so it is still in the $\mathrm{Y}$-Z plane. Therefore, the 
relationship between ECEF-OSC and ECI-OSC can be expressed by the rotation matrix around the $\mathrm{x}$-axis, i.e.,

$$
\mathbf{R}=\left[\begin{array}{ccc}
1 & 0 & 0 \\
0 & \cos \theta & \sin \theta \\
0 & -\sin \theta & \cos \theta
\end{array}\right]
$$

where $\theta$ is the rotation angle, the value can be determined by the following formula:

$$
\begin{aligned}
\cos \theta & =\left\langle\vec{v}_{\mathrm{ECEF}}(t), \hat{y}(t)\right\rangle /\left\|\vec{v}_{\mathrm{ECEF}}(t)\right\| \\
\sin \theta & =\left\langle\vec{v}_{\mathrm{ECEF}}(t), \hat{y}(t)\right\rangle /\left\|\vec{v}_{\mathrm{ECEF}}(t)\right\|
\end{aligned}
$$

Formula (5) can be calculated by establishing an auxiliary coordinate system, $O-X_{a} Y_{a} Z_{a}$, shown in Figure 4 . The subscript a stands for auxiliary. $X_{a}$ passes through the satellite ascending node, $Y_{a}$ pointing to the highest point of the orbit, and $Z_{a}$ being in the same direction as the angular velocity of the orbit. $\vec{n}_{s}$ and $\vec{n}_{E}$ are the angular velocities of the satellite and the Earth, respectively. $i_{c}$ is the orbital inclination of the master satellite.

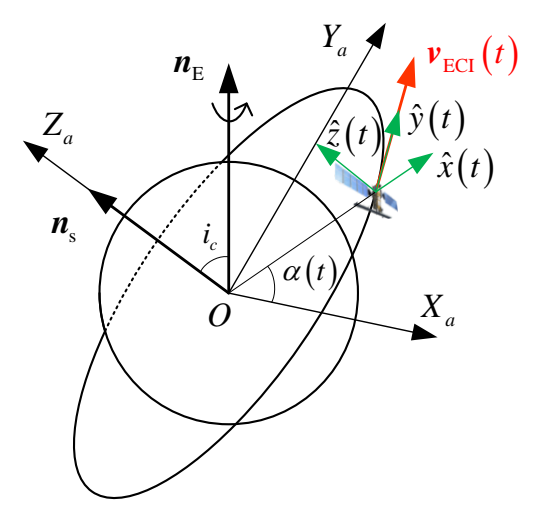

Figure 4. The auxiliary coordinate system.

It is easy to know that the direction of $z(t)$ is consistent with $\overrightarrow{O Z}_{a}$. In the auxiliary coordinate system, assuming that the argument of latitude (AOL) of the master satellite is $\alpha(t)$ (the angle from the ascending point to the current position), then:

$$
\alpha(t)=n_{s} t+M_{c}+\omega_{c}
$$

where $M_{c}$ and $\omega_{c}$ are the mean anomaly and argument of perigee (AOP) the master satellite, respectively.

From Figure 4, we have $\vec{v}(t)=\omega_{s} r(t) \cdot(\cos \alpha(t)$, $\sin \alpha(t), 0)$. $v(t)$ is also the direction of $y(t)$. So we have:

$$
\begin{aligned}
& \hat{x}=(\cos \alpha(t), \sin \alpha(t), 0) \\
& \hat{y}=(-\sin \alpha(t), \cos \alpha(t), 0) \\
& \hat{z}=(0,0,1)
\end{aligned}
$$

The angular velocity of the Earth rotation is $\vec{\omega}_{E}=\left(0, n_{E} \sin i_{c}, n_{E} \cos i_{c}\right)$. In the formula of the influence of the Earth rotation on the velocity, i.e., $\vec{v}_{E f f}(t)=\vec{v}(t)-\vec{\omega}_{E} \times \vec{r}(t)$, we have $\vec{r}(t)=r(t) \cdot \hat{x}$. Considering the case where the eccentricity of the master satellite is small, the variations of $r(t)$ and $v(t)$ during the entire orbit are negligible. So we can get:

$$
\begin{aligned}
& \left\langle\hat{y}, \vec{v}_{E f f}(t)\right\rangle \approx n_{s} r-\left\langle\vec{n}_{s}, \vec{n}_{E}\right\rangle r=\left(n_{s}-n_{E} \cos i_{c}\right) r \\
& \left\langle\hat{z}, \vec{v}_{E f f}(t)\right\rangle \approx n_{E} \sin i_{c} \cdot \cos \alpha(t) \cdot r
\end{aligned}
$$


Thus, we get:

$$
\begin{aligned}
& \cos \theta=\frac{n_{s}-n_{E} \cos i_{c}}{\sqrt{\left(n_{s}-n_{E} \cos i_{c}\right)^{2}+\left(n_{E} \sin i_{c} \cdot \cos \alpha(t)\right)^{2}}} \\
& \sin \theta=\frac{n_{E} \sin i_{c} \cdot \cos \alpha(t)}{\sqrt{\left(n_{s}-n_{E} \cos i_{c}\right)^{2}+\left(n_{E} \sin i_{c} \cdot \cos \alpha(t)\right)^{2}}}
\end{aligned}
$$

By substituting (10) into (4), the expression of the rotation matrix can be obtained.

Finally, the ECEF-RME can be expressed as:

$$
[\widetilde{x}(t), \widetilde{y}(t), \widetilde{z}(t)]^{\mathrm{T}}=\mathbf{R} \cdot[x(t), y(t), z(t)]^{\mathrm{T}}
$$

where the superscript $\mathrm{T}$ indicates transpose. For the detailed form, one can substitute (1) and (10) into (11).

To design formation SAR according to an application, we need to establish the relationship between baseline and orbital elements. Similar to (2), the baseline in the ECF coordinate system can be expressed as:

$$
\begin{aligned}
& B_{A T}(t)=|\widetilde{y}(t)| \\
& B_{\perp}(t)=\left|\bar{z}(t) \cos \theta_{d}-\widetilde{x}(t) \sin \theta_{d}\right|
\end{aligned}
$$

It is worth noting that the ECEF-RME determined by (1), (10) and (11) applies to LEO/MEO/GEO SAR, but we can simplify its form according to orbital altitude.

- In LEO SAR, $n_{S} \gg n_{E}$, so $n_{E}$ can be ignored. Thus, we have $\cos \theta \approx 1, \sin \theta \approx 0$, the rotation matrix is approximately the unit matrix, so the baseline calculated by ECEF-RME and ECI-RME is very close.

- In MEO SAR, the order of magnitude of $n_{S}$ and $n_{E}$ do not differ from each other considerably enough, so it is difficult to simplify the ECEF-RME further.

- In GEO SAR, the angular velocities of the Earth and the satellite cancel each other; instead, the rotation matrix is only determined by the inclination of the master satellite. In GEO SAR, $n_{S}=n_{E}$, (10) can be simplified as:

$$
\begin{aligned}
& \cos \theta=\tan \left(i_{c} / 2\right) / \sqrt{\tan ^{2}\left(i_{c} / 2\right)+\cos ^{2} \alpha(t)} \\
& \sin \theta=\cos \alpha(t) / \sqrt{\tan ^{2}\left(i_{c} / 2\right)+\cos ^{2} \alpha(t)}
\end{aligned}
$$

It is not difficult to imagine that, since the relative satellite-Earth motion, which is precisely the satellite's ECEF velocity, should be used for the spaceborne SAR focusing [33,34], the baseline calculation will also be based on the ECEF velocity. This is true for all spaceborne SAR, no matter what its orbit altitude is. In LEO SAR, the influence of Earth rotation is slight, and directions of satellite's ECEF and ECI velocities are very close to each other, so the baseline calculated by ECI-RME is of high accuracy. However, in GEO SAR, the influence of Earth rotation is significantly more severe, resulting in an increased difference between the satellite's ECEF and ECI velocity directions [35,36]; therefore, using ECI-RME to calculate the baseline will lead to significant errors.

It can be seen that the rotation matrix is time-varying, which results in the ECEF-RME not having a regular geometry. Therefore it is challenging to adopt the geometry parameter-based design method used for ECI-RME. To address this issue, we propose a numerical formation design method based on the minimum baseline error criterion. For a specific application, we usually require the along-track baseline and the perpendicular baseline as stable as possible. Assuming that the objective along-track baseline and perpendicular baseline are $B_{0 A T}$ and $B_{0 \perp}$, respectively, the average baseline error can be expressed as:

$$
C=\int_{0}^{T}\left[\left(B_{\perp}^{2}(t)-B_{0 \perp}^{2}\right)^{2}+\left(B_{A T}^{2}(t)-B_{0 A T}^{2}\right)^{2}\right] d t
$$


where $T$ is the orbital period. The formation design method based on the minimum baseline error criterion is to minimize the average baseline error shown in (14). Using a numerical optimization method, one can obtain the orbital elements of the slave satellite.

\section{GEO InSAR Formation Design Based on MABC}

An obvious way to obtain a SAR formation with relatively stable baselines is to minimize the error shown in (13) by numerical optimization. This method, however, has two disadvantages: (1) the computation complexity is considerable; (2) it is difficult to find out a general rule of formation that satisfies the performance requirements. In this section, we propose a GEO InSAR formation design method based on the Minimum Along-track Baseline Criterion (MABC). According to this method, the analytical expression of the orbital elements of the slave satellite in GEO InSAR formation can be obtained.

According to (13), we can get the ECEF-RME in GEO SAR formation as:

$$
\begin{aligned}
& \widetilde{x}(t)=x(t) \\
& \widetilde{y}(t)=\frac{\tan \left(i_{c} / 2\right) \cdot y(t)+\cos \alpha(t) \cdot z(t)}{\sqrt{\tan ^{2}\left(i_{c} / 2\right)+\cos ^{2} \alpha(t)}} \\
& \widetilde{z}(t)=\frac{-\cos \alpha(t) \cdot y(t)+\tan \left(i_{c} / 2\right) \cdot z(t)}{\sqrt{\tan ^{2}\left(i_{c} / 2\right)+\cos ^{2} \alpha(t)}}
\end{aligned}
$$

Considering that the off-nadir angle of satellite in GEO SAR is very small, generally $\theta_{d}=1.5^{\circ} \sim$ $7.5^{\circ}$, it can be ignored when calculating baseline according to (12), i.e., the perpendicular baseline is almost determined by the z-axis component in ECEF-RME, shown as:

$$
\mid \begin{aligned}
& B_{A T}(t)|=| \widetilde{y}(t) \mid \\
& B_{\perp}(t)|\approx| z(t) \mid
\end{aligned}
$$

The perpendicular baseline affects the height measurement accuracy of InSAR, which should be better able to be kept stable. On the other hand, to reduce the rotation decorrelation introduced by the along-track baseline [3], we need to keep the baseline along the orbit as small as possible. That is to say, in the average baseline error shown in (14), we need to set an objective perpendicular baseline $B_{0 \perp}$ according to height retrieval accuracy requirement and an objective along-track baseline $B_{0 A T}=0$.

It is noted that the rotation matrix has nothing to do with the orbital elements of the slave satellite. Therefore, like the ECI-RME, we need to design variables $A_{1}, A_{2}, l_{1}, \alpha_{1}$ and $\alpha_{2}$. In Appendix $\mathrm{A}$, we use the combination of simulation verification and theoretical derivation to point out that according to the average baseline error shown in (14), we can get $A_{1}=0, \alpha_{2}=0$ or $\pi$; therefore, we can derive the results that the orbital elements of the master and slave satellite meet $\Delta e=0, \Delta M=0$ and $\Delta i=0$. Generally, the master and slave satellites have the same semi-major axis. So we only need to design the RAAN and AOP of the slave satellite.

In the following, we will deduce the analytical expression of the orbital elements of slave satellite in GEO InSAR formation by reasonably modifying the optimization problem. Based on the results of $\Delta e=0, \Delta M=0$ and $\Delta i=0$, the baseline of the formation can be further simplified to:

$$
\begin{aligned}
& \widetilde{y}(t)=\frac{a\left\{\Delta \omega \tan \left(i_{c} / 2\right)-\Delta \Omega \sin \left(i_{c}\right) / 2 \cdot\left[\tan ^{2}\left(i_{c} / 2\right)+\cos (2 n t)\right]\right\}}{\sqrt{\tan ^{2}\left(i_{c} / 2\right)+\cos ^{2}(n t)}} \\
& \widetilde{z}(t)=-a[\Delta \omega+\Delta \Omega] \underbrace{\frac{\cos (n \cdot t)}{\sqrt{\tan ^{2}\left(i_{c} / 2\right)+\cos ^{2}(n \cdot t)}}}_{s(t)}
\end{aligned}
$$

It should be noted that the shape of the perpendicular baseline in the whole orbit, denoted as $s(t)$, is independent of the orbit elements of the slave satellite, but only related to the inclination of the master satellite. The sum of RAAN difference $\Delta \Omega$ and AOP difference $\Delta \omega, \Delta \omega+\Delta \Omega$, only affect the 
amplitude of the perpendicular baseline. Figure 5 shows the shape of the perpendicular baseline in the whole orbit, where 3 inclinations are selected. However, the relationship between along-track baseline and orbit elements difference $\Delta \Omega$ and $\Delta \omega$ is much more complicated, shown as (17).

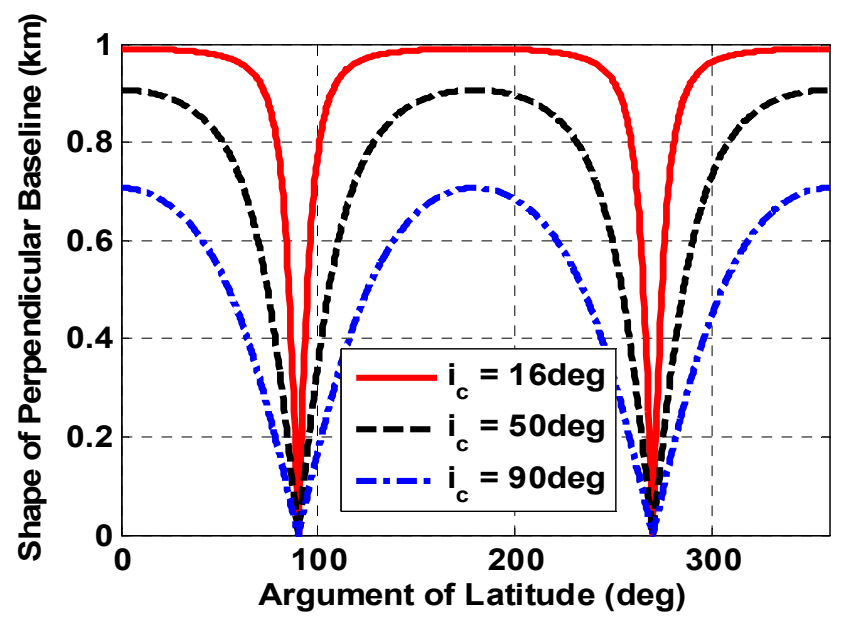

Figure 5. Shape of perpendicular baseline for different inclinations in GEO SAR.

Because the perpendicular baseline and the along-track baseline vary throughout the whole orbit, we propose the minimum along-track baseline criterion under the root mean square (RMS) constraint of the perpendicular baseline. The objective of the criterion is to minimize the RMS of the along-track baseline, on the premise that the RMS of the perpendicular baseline is equal to the design requirement $B_{0 X T}$. The optimization problem can be expressed as:

$$
\begin{aligned}
(\Delta \omega, \Delta \Omega)= & \underset{\Delta \omega, \Delta \Omega}{\arg \min } \int \widetilde{y}^{2}(t) d t \\
\text { s.t. } E & \left\{\widetilde{z}^{2}(t)\right\}=B_{0 X T}^{2}
\end{aligned}
$$

Under the constraint of $E\left\{\bar{z}^{2}(t)\right\}=B_{0 X T}^{2}$, let the derivative of $\int \widetilde{y}^{2}(t) d t$ for $\Delta \omega$ and $\Delta \Omega$ be zero, the orbital elements of the slave satellite can be obtained, which will be shown in the following.

From $E\left\{\bar{z}^{2}(t)\right\}=B_{0 X T}^{2}$ we have:

$$
a^{2}(\Delta \omega+\Delta \Omega)^{2}\left(1-\frac{\tan \left(i_{c} / 2\right)}{\sqrt{1+\tan ^{2}\left(i_{c} / 2\right)}}\right)=B_{0 X T}^{2}
$$

Thus, $|\Delta \omega+\Delta \Omega|=B_{0 X T} /\left[a \sqrt{1-\sin \left(i_{c} / 2\right)}\right]$. Denote $\Delta \omega+\Delta \Omega=C_{1}$, where $C_{1}=$ $\pm B_{0 X T} /\left[a \sqrt{1-\sin \left(i_{c} / 2\right)}\right]$. Suppose:

$$
\begin{aligned}
& \Delta \Omega=\mu \cdot C_{1} \\
& \Delta \omega=(1-\mu) \cdot C_{1}
\end{aligned}
$$

then:

$$
\widetilde{y}(t)=\frac{a C_{1} \tan \left(i_{c} / 2\right)}{\sqrt{\tan ^{2}\left(i_{c} / 2\right)+\cos ^{2}(n t)}}\left\{1-\frac{2 \mu\left[\tan ^{2}\left(i_{c} / 2\right)+\cos ^{2}(n t)\right]}{1+\tan ^{2}\left(i_{c} / 2\right)}\right\}
$$

Ignoring the constant $a C_{1} \tan \left(i_{c} / 2\right)$, and denoting $v=\tan \left(i_{c} / 2\right), u=n t, C_{2}=2 /\left[1+\tan ^{2}\left(i_{c} / 2\right)\right]$, we can obtain:

$$
\vec{y}^{2}(u)=\frac{1}{v^{2}+\cos ^{2} u}-2 \mu C_{2}+C_{2}^{2} \mu^{2}\left(v^{2}+\cos ^{2} u\right)
$$


In this way, only one undetermined parameter $\mu$ needs to be solved in the expression of integration of along-track baseline as:

$$
\int_{0}^{2 \pi} \vec{y}^{2}(u) d u=-2 \mu C_{2} \cdot 2 \pi+C_{2}^{2} \mu^{2} v^{2} \cdot 2 \pi+C_{2}^{2} \mu^{2} \pi+\int_{0}^{2 \pi} \frac{1}{v^{2}+\cos ^{2} u} d u
$$

By finding the derivative of $\int \widetilde{y}^{2}(u) d u$ for $\mu$ and letting the derivative be 0 , we can get the $\mu$ value that makes $\int \widetilde{y}^{2}(u) d u$ the smallest. From $\partial \int_{0}^{2 \pi} \widetilde{y}^{2}(u) d u / \partial \mu=0$ we can obtain:

$$
\mu=\frac{2}{C_{2}\left(2 v^{2}+1\right)}=\frac{\tan ^{2}\left(i_{c} / 2\right)+1}{2 \tan ^{2}\left(i_{c} / 2\right)+1}
$$

Thus, the orbital elements of the slave satellite are:

$$
\begin{aligned}
& e_{d}=e_{c} \\
& i_{d}=i_{c} \\
& M_{d}=M_{c} \\
& \Omega_{d}=\Omega_{c}+\mu C_{1} \\
& \omega_{d}=\omega_{c}+(1-\mu) C_{1}
\end{aligned}
$$

where

$$
\begin{aligned}
& C_{1}= \pm \frac{B_{0 X T}}{a \sqrt{1-\sin \left(i_{c} / 2\right)}} \\
& \mu=\frac{\tan ^{2}\left(i_{c} / 2\right)+1}{2 \tan ^{2}\left(i_{c} / 2\right)+1}
\end{aligned}
$$

According to the design requirements of the perpendicular baseline $B_{0 X T}$, the orbital inclination $i_{c}$ of the master satellite, we can get the orbital elements of the slave satellite for GEO InSAR from (25) and (26).

\section{Simulation}

\subsection{Simulation Settings}

To verify the validity of the derived ECEF-RME and the analytical expression of orbital elements obtained by the proposed MABC for GEO InSAR formation design, we select typical orbital elements of the LEO/MEO/GEO SAR for simulation. The sub-satellite point trajectory is shown in Figure 6. The off-nadir angles are $35.79^{\circ}, 7.85^{\circ}$, and $5.57^{\circ}$, respectively, so that the incident angle is $40^{\circ}$. The satellites use 2D attitude control to achieve zero Doppler centroid [35].

The simulation includes three parts. The first part verifies the accuracy of the ECEF-RME in LEO, MEO, and GEO SAR. The second part verifies the derived analytical expression of orbital elements and compares the design result with the numerical optimization of (14). The third part simulates InSAR processing using the orbital elements derived from the classical ECI-RME and the proposed formation design method and compares their results. Please note that the orbital elements of the slave satellite in GEO SAR listed in Table 1 are given arbitrarily and adopted only to verify the accuracy of ECEF-RME, as will be discussed in Section 5.2. The elements suitable for GEO InSAR will be calculated according to the analytical expression, shown in Section 5.3. The parameters for InSAR simulation are listed in Table 2, and the formation operates above the equator. In the simulation, the satellites' positions and velocities come from the Satellite Tool Kit (STK) by Analytical Graphics, Inc. from the United States. The STK's high-precision orbit prediction (HPOP) mode provides precise satellites' positions according to the orbital elements. This can be served as the verification data for the proposed methods. 


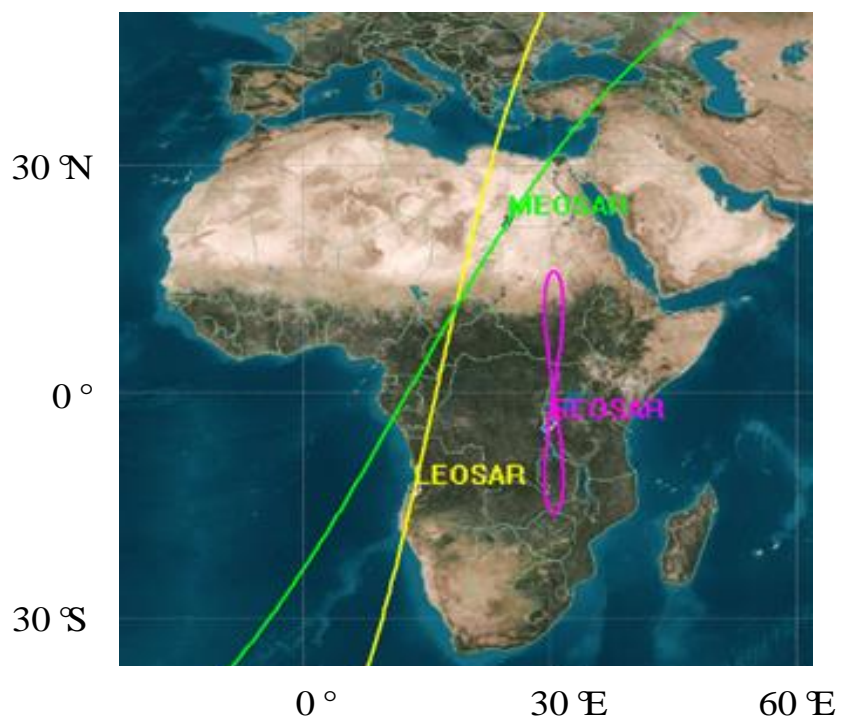

Figure 6. Nadir-point tracks of LEO SAR, MEO SAR, and GEO SAR (by STK).

Table 2. Parameters for InSAR processing simulation.

\begin{tabular}{cccc}
\hline Parameters & Value & Parameters & Value \\
\hline Wavelength $(\mathrm{cm})$ & 24 & Incidence angle $\left(^{\circ}\right)$ & 30 \\
Bandwidth $(\mathrm{MHz})$ & 18 & Number of looks & $5 \times 5$ \\
Pulse width $(\mu s)$ & 20 & SNR $(\mathrm{dB})$ & 10 \\
PRF $(\mathrm{Hz})$ & 100 & Integration time $(\mathrm{s})$ & 320 \\
\hline
\end{tabular}

It is noted that the rotation matrix has nothing to do with the orbital elements of the slave satellite. Therefore, like the ECI-RME, we need to design variables $A_{1}, A_{2}, l_{1}, \alpha_{1}$ and $\alpha_{2}$. In Appendix A, we use the combination of simulation verification and theoretical derivation to point out that according to the average baseline error shown in (14), we can get $A_{1}=0, \alpha_{2}=0$ or $\pi$; therefore, we can derive the results that the orbital elements of the master and slave satellite meet $\Delta e=0, \Delta M=0$ and $\Delta i=0$. Generally, the master and slave satellites have the same semi-major axis, so we only need to design the RAAN and AOP of the slave satellite.

\subsection{Verification of ECEF-RME}

Under the above simulation parameters, the baseline obtained using the derived ECEF-RME is shown in Figure 7. The "STK Data" line is the baseline calculated according to the satellites' positions obtained from STK, which is regarded to be accurate as a contrast. We also calculate the baseline according to ECI-RME, in which the results of ECEF-RME are compared.

Since the absolute size of baselines in different orbital altitudes are quite different, to evaluate the accuracy of the baseline calculated with ECI-RME and ECEF-RME and compare them in different orbital altitudes, we introduce a normalized baseline error as:

$$
\eta=\frac{1}{T} \int_{0}^{T} \frac{\sqrt{\Delta B_{A T}^{2}(t)+\Delta B_{\perp}^{2}(t)}}{|B(t)|} d t
$$

where $\Delta B_{A T}(t), \Delta B_{\perp}(t)$ represent the error of along-track baseline and error of perpendicular baseline, respectively. The error is calculated compared with the "STK Data". $|B(t)|=\sqrt{\bar{x}^{2}(t)+\widetilde{y}^{2}(t)+\widetilde{z}^{2}(t)}$ represents the length of the spatial baseline using "STK Data". The normalized baseline error of the ECEF-RME and ECI-RME in for each orbital height is shown in Table 3. 

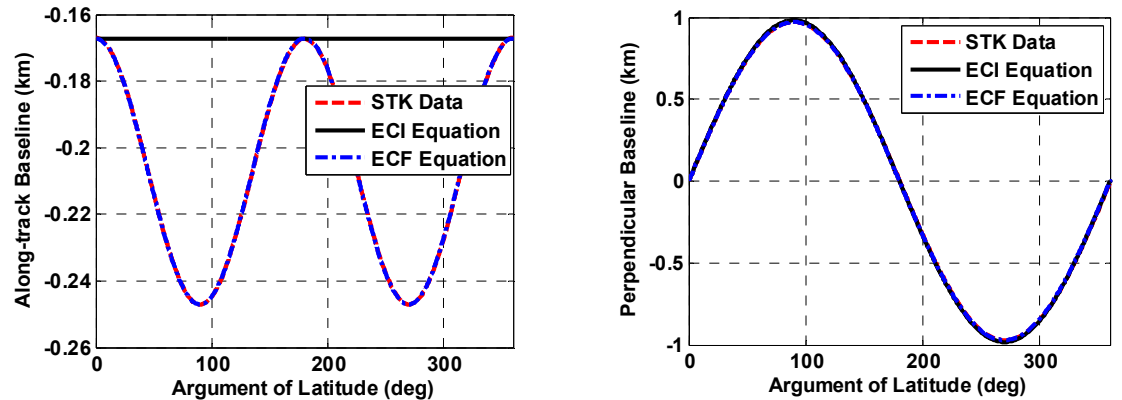

(a)
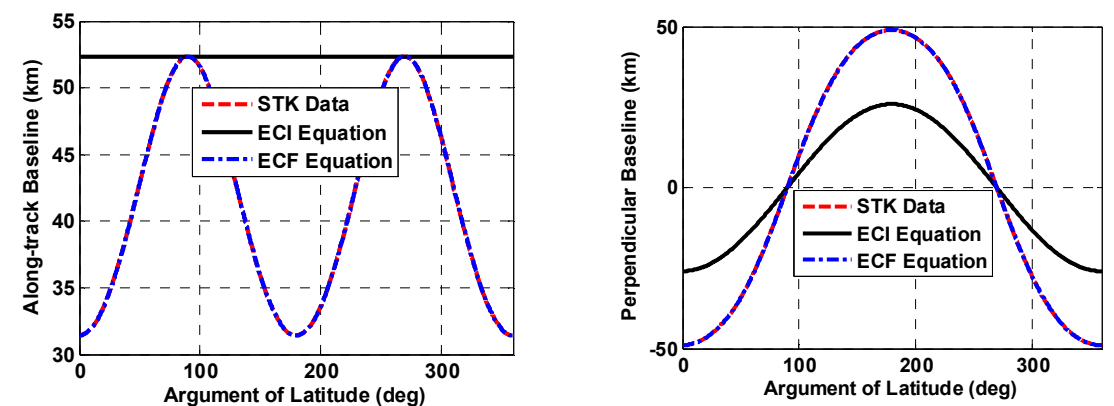

(b)
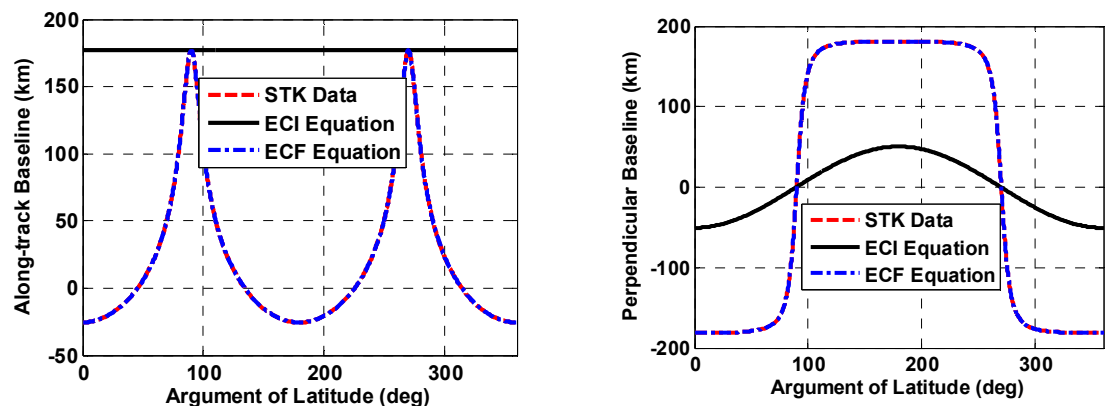

(c)

Figure 7. Baseline calculated using ECF equation, ECI equation, and STK data. (a) LEO SAR formation; (b) MEO SAR formation; (c) GEO SAR formation.

Table 3. Normalized baseline error using ECEF-RME or ECI-RME in LEO SAR, MEO SAR, and GEO SAR.

\begin{tabular}{ccc}
\hline & $\eta_{\text {ECF }}$ & $\eta_{\text {ECI }}$ \\
\hline LEO SAR & $0.54 \%$ & $4.4 \%$ \\
MEO SAR & $0.056 \%$ & $35 \%$ \\
GEO SAR & $0.046 \%$ & $115 \%$ \\
\hline
\end{tabular}

It can be seen that as the orbital altitude increases, the normalized error using ECI-RME increases, while the ECEF-RME always has high accuracy. Besides, it is noted that in LEO SAR, the error of ECEF-RME is higher than MEO SAR and GEO SAR. This is because the LEOSAR satellite simulated here has a certain eccentricity. Since the RME itself is derived on the premise of small orbital eccentricity [31], the accuracy of both ECI-RME and ECEF-RME will decrease as the eccentricity increases.

\subsection{Verification of GEO InSAR Formation Design Method}

It is noted that in the derivation of GEO InSAR formation design method, we obtained consistent orbital elements of the slave satellite with the main satellite by simulating the baseline error shown 
in (14). In the subsequent process, to obtain the analytical expression of the orbital elements, we made a reasonable modification to the optimization problem. Therefore, the orbital elements obtained by the analytical expression and the numerical method will be a bit different. In this sub-section, we first verify that the formation obtained by the analytical expression satisfies the perpendicular baseline requirements of InSAR, and secondly compare the along-track baseline difference between the formations obtained by the numerical method and the analytic expression.

Using the parameters in Table 2, the optimal baseline [28] can be calculated as $136 \mathrm{~km}$. Setting $B_{0 \perp}=136 \mathrm{~km}$, the orbital elements of the GEO SAR master satellite are used as shown in Table 1, then according to (25) and (26), the orbital elements of the slave satellite can be obtained as $\Omega_{d}= \pm 0.1831^{\circ}$, $\omega_{d}= \pm 3.546 \times 10^{-3 \circ}$. By minimizing the baseline error shown in (14) using the numerical method, the orbital elements of the slave satellite are $\Omega_{d}=-0.3131^{\circ}, \omega_{d}=0.1262^{\circ}$.

The baseline of the formations obtained by the numerical optimization method and the analytical expression, respectively, are shown in Figure 8. It can be found that for both methods, the peak value of the perpendicular baseline is very close to the design requirements, which are $136 \mathrm{~km}$ and $136.2 \mathrm{~km}$ for analytical expression and numerical method, respectively; for the along-track baseline, there exist orbital positions where one method outperforms the other. However, near the orbital position where the perpendicular baseline reaches a peak value, the numerical optimization method has a much larger along-track baseline, $45.0 \mathrm{~km}$, which is more than twice that in the analytical expression, $18.4 \mathrm{~km}$. Therefore, the numerical optimization method will introduce a more severe rotation decorrelation near these orbital positions. These analyses show that the InSAR formation obtained by the proposed analytical design method, shown in (26), not only satisfies the optimal perpendicular baseline design requirements but can also provide SAR image pairs with a higher correlation coefficient than the numerical optimization method.

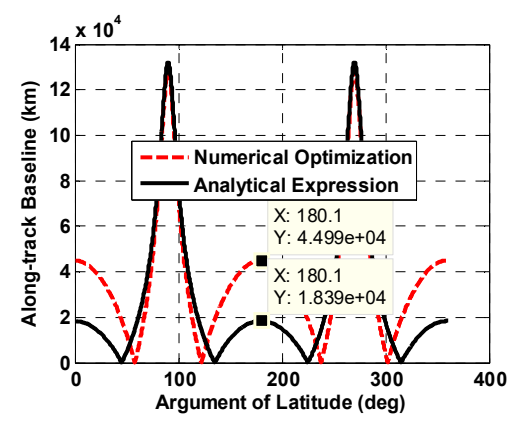

(a)

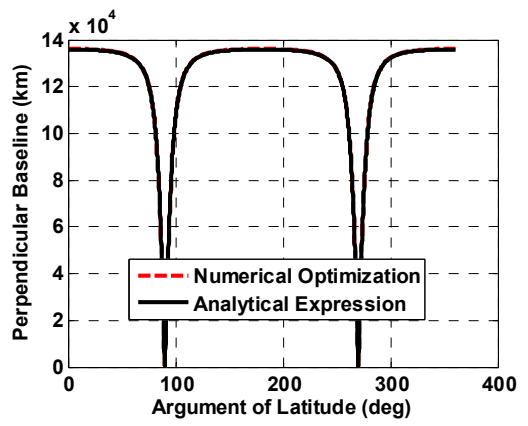

(b)

Figure 8. Baseline comparison of numerical optimization and proposed analytical expression for GEO InSAR. (a) Along-track baseline; (b) Perpendicular baseline.

As can be seen from both Figures 5 and 8, the GEO InSAR always has moments where the perpendicular baseline is equal to zero. This shows that no matter how we design the orbital elements, it is impossible to obtain perpendicular baselines that are always greater than zero or less than zero. After using the proposed formation design method for GEO InSAR, the perpendicular baseline is a near-periodic function whose period is half that of the orbital period. Therefore, the perpendicular baseline will have two zero points throughout the whole orbit.

In fact, we can theoretically prove through the counter-evidence method that the perpendicular baseline has at least two zero points, no matter it has undergone a formation design or not. See Appendix B for the detailed process.

\subsection{Comparison of InSAR Results between the Proposed Method and ECI-RME-Based Method}

In this sub-section, we compare InSAR processing and results using the orbital elements derived from the classical ECI-RME and the proposed formation design method. First of all, we give a brief 
derivation of the ECI-RME-based design results. When the ECI-RME is used to design GEO InSAR formation, the vertical baseline is very close to the $\mathrm{z}$-direction baseline due to the small off-nadir angle of GEO SAR. The baseline in the z-direction is a cosine function, so again we use the method of mean optimization, that is, $E\left\{z^{2}(t)\right\}=B_{0 X T}^{2}$. On the other hand, the along-track baseline (y-direction) is completely decoupled from the vertical baseline (i.e., independently determined by different orbital elements), so we can set the along-track baseline to be zero. Furthermore, since the effective velocity of GEO SAR above the equator reaches the peak value during the whole orbit, its corresponding integration time will be the least. So we consider that the vertical baseline above the equator reaches the peak. It is easy to obtain the expression of the slave satellite's orbital elements according to these conditions, and the results are $\Delta a=0, \Delta e=0, \Delta M=0, \Delta i=0, \Delta \Omega= \pm \sqrt{2} B_{0 X T} /\left(a \sin i_{c}\right)$, $\Delta \omega=\mp \sqrt{2} B_{0 X T} /\left(a \tan i_{c}\right)$. Using the master satellite's orbital elements, shown in Table 1 , the design results are $\Delta \Omega=-0.9482^{\circ}, \Delta \omega=0.9115^{\circ}$. The positions of the satellites designed by the two methods are shown in Figure 9a,b. It can be seen that the along-track baseline obtained by the proposed method is very small while using the ECI-RME-based method will lead to a considerable deviation between the vertical baseline and the design value. Besides, the along-track baseline is not zero as desired.

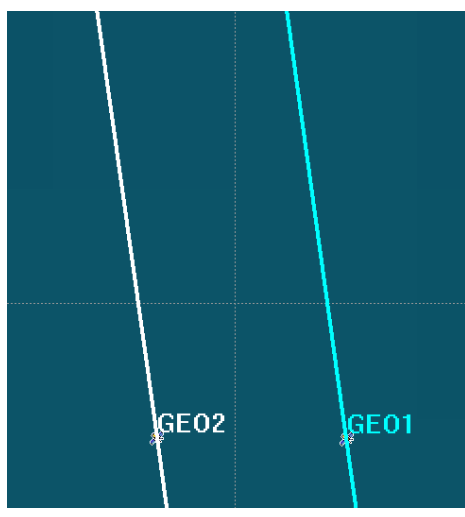

(a)

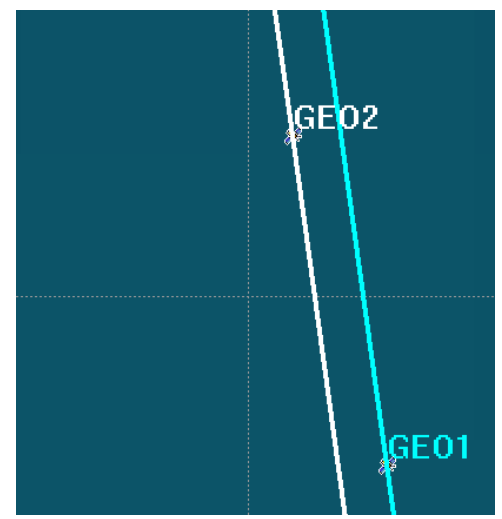

(b)

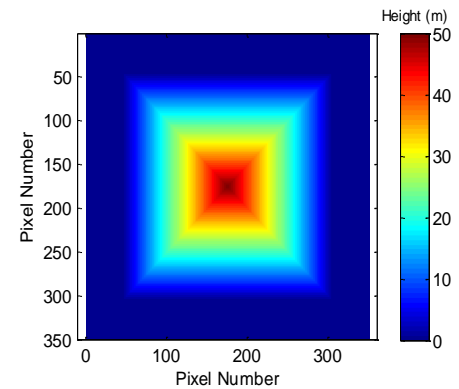

(c)

Figure 9. Formation design results and DEM setting. (a) Formation designed by the proposed method; (b) Formation designed according to ECI-RME; (c) DEM setting for simulation.

We set up a scene with a size of $3.5 \mathrm{~km} \times 3.5 \mathrm{~km}$, in the middle of which a $2.6 \mathrm{~km} \times 2.6 \mathrm{~km}$ pyramid with a height of $50 \mathrm{~m}$ is set. The DEM settings of the scene are shown in Figure 9c, and the DEM inversion results of the scene are shown in Figure 10. It can be seen that the proposed method has the highest retrieval accuracy, which is $0.67 \mathrm{~m}$ (mean root square error). For the formation designed by the ECI-RME-based method, the perpendicular baseline is relatively short, at $34 \mathrm{~km}$. However, if the data is not optimally selected, the retrieval accuracy could be very poor due to the low correlation coefficient (0.33). After applying the optimal data acquisition based on the minimum rotation decorrelation [3], the correlation coefficient is much improved, reaching 0.90 ; but the accuracy, which is $1.01 \mathrm{~m}$, is still not as good as the proposed method. This is because the perpendicular baseline is not the desired optimal one. Additionally, after the optimal data acquisition, the aperture center time difference between the satellites is $222 \mathrm{~s}$. That is to say, the acquisition times of the image pair have a considerable interval, which will deteriorate the formation's performance for eliminating the atmospheric disturbance. 


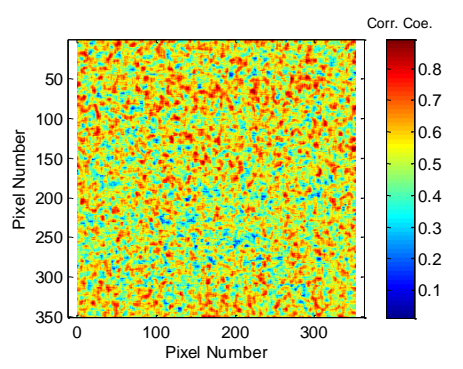

(a)

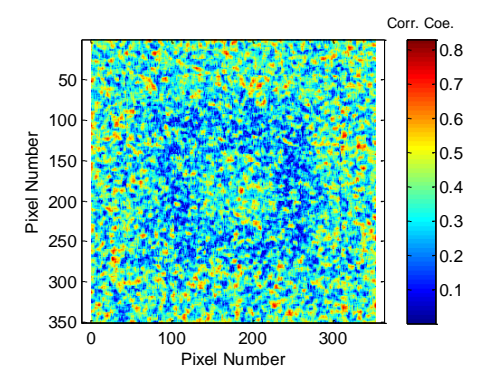

(d)

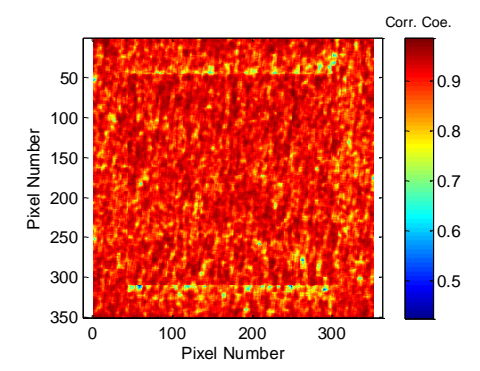

(g)

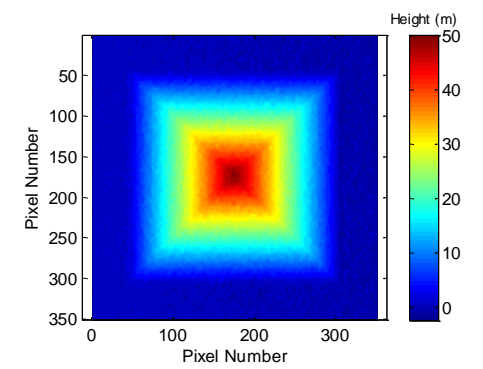

(b)

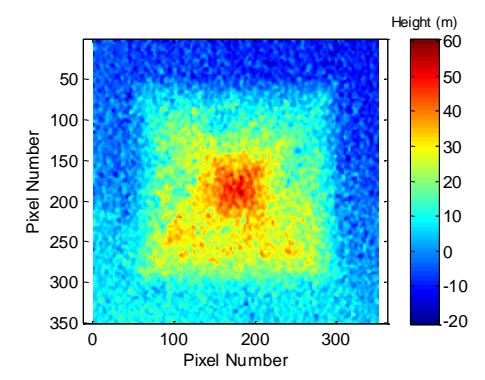

(e)

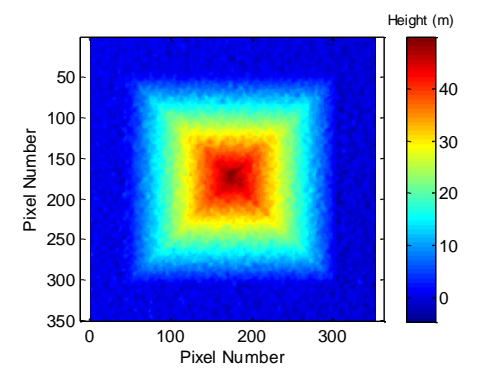

(h)

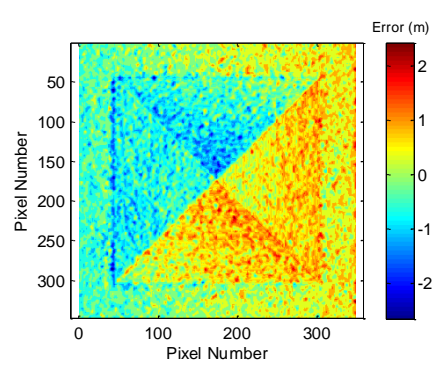

(c)

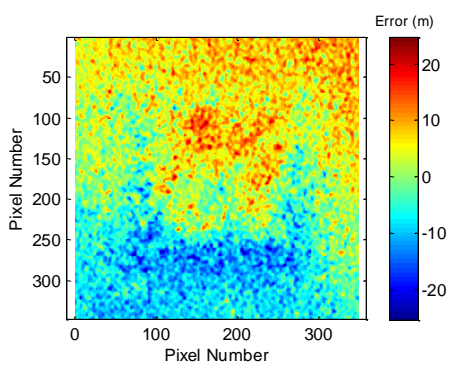

(f)

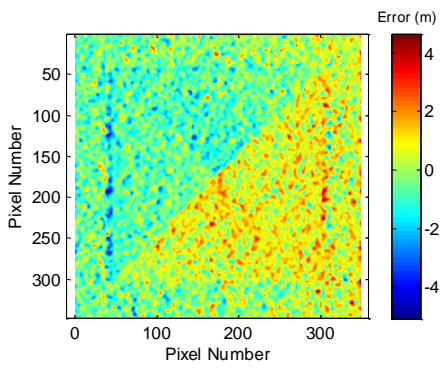

(i)

Figure 10. InSAR simulation results. (a) The correlation coefficient (corr. coe.), with a mean value of 0.53 ; (b) Retrieved height; (c) Retrieval error, with a mean value of $0.67 \mathrm{~m}$ of the SAR pairs acquired from the formation by the proposed method; (d) Corr. coe., with a mean value of 0.33 ; (e) Retrieved height; (f) Retrieval error, with a mean value of $7.62 \mathrm{~m}$ of the SAR pairs acquired from the formation by ECI-RME, without optimal data acquisition; (g) Corr. coe., with a mean value of 0.90; (h) Retrieved height; (i) Retrieval error, with a mean value of $1.01 \mathrm{~m}$ of the SAR pairs acquired from the formation by ECI-RME, the optimal data acquisition was conducted.

\section{Discussion}

The proposed method can obtain a vertical baseline approaching the optimal baseline as much as possible and reduce the along-track baseline as much as possible, so it has the following advantages in InSAR applications:

- Shorten the acquisition time interval between master and slave images, to effectively reduce the atmospheric impact. When applying the ECI-RME to design the formation, a relatively large along-track baseline will occur, decreasing the correlation coefficient. Thus, it is necessary to use the minimum rotation decorrelation-based method to select the optimal data for imaging. However, the acquisition time interval between master and slave images will hence enlarge, which will make it difficult to eliminate the time-varying atmospheric impact. The proposed method can achieve near-real-time baseline to reduce the impact of the atmosphere.

- Improve the accuracy of DEM retrieval. A large part of the vertical baseline obtained by using the ECI-RME-based method is transformed into the along-track baseline because the satellite's 
effective velocity direction changed much due to the Earth's rotation, so it is far from the design requirements. By considering the Earth's rotation, the formation designed by the proposed method can be closer to the optimal vertical baseline, to achieve higher accuracy of DEM retrieval.

- Improve coverage performance and reduce beam control requirements of the satellite platform. Because the ECI-RME-based method will bring obvious along-track baseline, if both the master and slave satellites use the same beam control scheme, the azimuth overlap rate of the beam will be reduced. The proposed method can eliminate the along-track baseline so that the master and slave satellites can achieve a higher beam overlap rate while using the same beam control scheme.

\section{Conclusions}

In this paper, the Relative Motion Equation under ECEF coordinate system (ECEF-RME) is obtained based on the Coordinate Rotational Transformation, which can accurately calculate the baseline of the spaceborne SAR formation for all orbital altitude. The ECEF-RME no longer has significant geometry features, so it is challenging to design a formation using the geometry parameters as ECI-RME does. To address this issue, we can obtain the orbital elements by minimizing the mean square root of the baseline proposed in this paper, using a numerical optimization method. In GEO InSAR, we only need to design the RAAN and AOP of the slave satellite, whose analytical expressions are exclusively related to the inclination angle of the master satellite and the perpendicular baseline requirement. The GEO InSAR formation obtained by this analytical expression meets the perpendicular baseline requirements and has a small along-track baseline, and thus can improve the correlation coefficient of the interferogram. This method can realize a real-time vertical baseline, thus can alleviate the atmospheric disturbance and help improve the DEM retrieval accuracy.

Author Contributions: Conceptualization, Z.C.; Methodology, Z.C.; Validation, X.D.; Supervision, Y.L.; Writing-Original Draft, Z.C. and X.D.; Writing-Review \& Editing, C.H. All authors have read and agreed to the published version of the manuscript.

Funding: This research was funded in part by the National Natural Science Foundation of China under Grant Nos. 61960206009, 61971039, National Ten-thousand Talents Program 'Young top talent' (Grant No. W03070007), the Special Fund for Research on National Major Research Instruments (NSFC Grant Nos. 61827901, 31727901), and the Young Elite Scientists Sponsorship Program by CAST (2017QNRC001).

Acknowledgments: The authors would like to thank the editors and reviewers for the constructive comments which helped improve this paper significantly and made it clearer.

Conflicts of Interest: The authors declare no conflict of interest. The funders had no role in the design of the study; in the collection, analyses, or interpretation of data; in the writing of the manuscript, or in the decision to publish the results.

\section{Appendix A}

In this Appendix, we present partial simulation and theoretical verification of ECEF-RME simplification in the GEO InSAR formation design. Through numerical simulation, we can find that when $\alpha_{1}$ and $\alpha_{2}$ are fixed, $A_{1}=0$ is always true when the baseline error shown in (14) is minimum. Figure A1 shows the simulation of baseline error under some parameters, where $B_{0 X T}=150$.

In theory, we can verify that $A_{1}=0$ is a solution of $\partial C / \partial A_{1}=0$. This will partially support the conclusion that $A_{1}=0$. The verification is as follows.

Denote

$$
\left\{\begin{array}{l}
\cos \beta(t)=\cos \alpha(t) / \sqrt{\tan ^{2}\left(i_{c} / 2\right)+\cos ^{2} \alpha(t)} \\
\sin \beta(t)=\tan \left(i_{c} / 2\right) / \sqrt{\tan ^{2}\left(i_{c} / 2\right)+\cos ^{2} \alpha(t)}
\end{array}\right.
$$

then

$$
\begin{gathered}
\widetilde{y}(t)=\sin \beta(t) y(t)+\cos \beta(t) z(t) \\
\widetilde{z}(t)=-\cos \beta(t) y(t)+\sin \beta(t) z(t)
\end{gathered}
$$


Please note that $\sin \beta(t+T / 2)=\sin \beta(t)$ (called "even function"), $\cos \beta(t+T / 2)=-\cos \beta(t)$ (called "odd function"). Therefore, $\widetilde{y}(t)$ and $\widetilde{z}(t)$ can be written as the sum of the odd function and the even function, respectively, i.e.,

$$
\begin{aligned}
& \widetilde{y}(t)=\underbrace{\left[A_{1} \sin \left(n t+\alpha_{1}\right) \sin \beta(t)\right]}_{y o}+\underbrace{\left[l_{1} \sin \beta(t)+A_{2} \cos \beta(t) \cos \left(n t+\alpha_{2}\right)\right]}_{\text {ye }} \\
& \widetilde{z}(t)=\underbrace{\left[-l_{1} \cos \beta(t)+A_{2} \sin \beta(t) \cos \left(n t+\alpha_{2}\right)\right]}_{z o}+\underbrace{\left[-A_{1} \cos \beta(t) \sin \left(n t+\alpha_{1}\right)\right]}_{z e}
\end{aligned}
$$

where $y o, y e, z o$ and ze represents the odd and even components of $\widetilde{y}(t)$ and $\widetilde{z}(t)$. Therefore

$$
\begin{aligned}
& \int \widetilde{y}^{2}(t) d t=\int(y o)^{2}+(y e)^{2} d t \\
& \int \widetilde{z}^{2}(t) d t=\int(z o)^{2}+(z e)^{2} d t \\
& \int \widetilde{y}^{4}(t) d t=\int(y o)^{4}+(y e)^{4}+6(y o)^{2}(y e)^{2} d t \\
& \int \widetilde{z}^{4}(t) d t=\int(z o)^{4}+(z e)^{4}+6(z o)^{2}(z e)^{2} d t
\end{aligned}
$$

Thus, the orders of $A_{1}$ are all no less than two, which means the derivative of $C$ w.r.t. $A_{1}$ is at least the first-order term of $A_{1}$, so $A_{1}=0$ is a solution of $\partial C / \partial A_{1}=0$. From $A_{1}=0$ we can obtain $e_{c}=e_{d}$ and $\Delta M=0$. Therefore, there are three independent variables $l_{1}, A_{2}$, and $\alpha_{2}$ left in the baseline error expression.

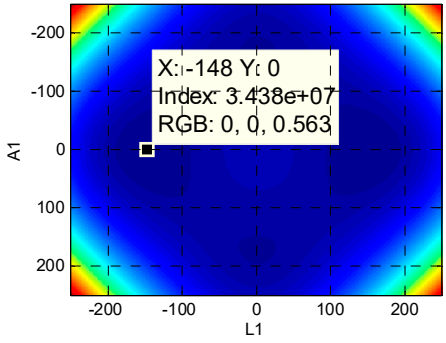

(a)

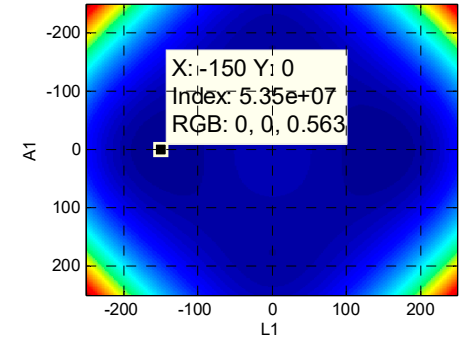

(b)

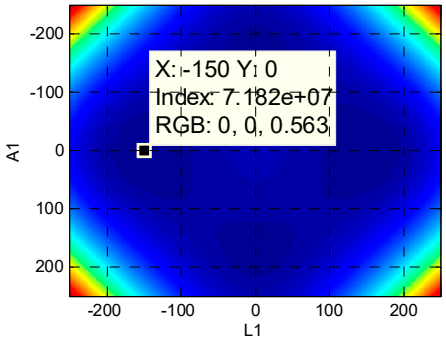

(c)

Figure A1. Part of the simulation verification of numerical optimization with fixed $\alpha_{1}$ and $\alpha_{2}$. The corresponding parameters are: (a) $\alpha_{1}=30^{\circ}, \alpha_{2}=20^{\circ}, i_{c}=8^{\circ}$; (b) $\alpha_{1}=0^{\circ}, \alpha_{2}=90^{\circ}, i_{c}=12^{\circ}$; (c) $\alpha_{1}=90^{\circ}, \alpha_{2}=90^{\circ}, i_{c}=16^{\circ}$.

It can be seen from a further simulation that when $A_{2}$ is a fixed value, the minimum baseline error can be obtained at $\alpha_{2}=0$ or $\pi$. Figure A2 shows the baseline error under some parameters, where $B_{0 X T}=150$. According to $\alpha_{2}=0$ or $\pi$ we can get $\eta=0$ or $\pi$, and then get $\Delta i=0$.

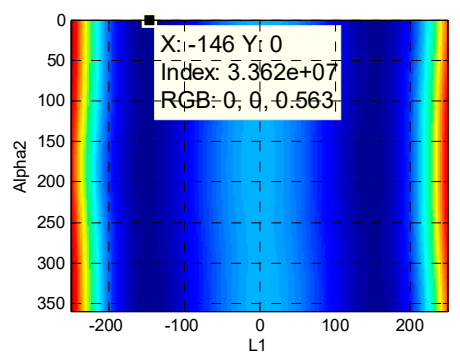

(a)

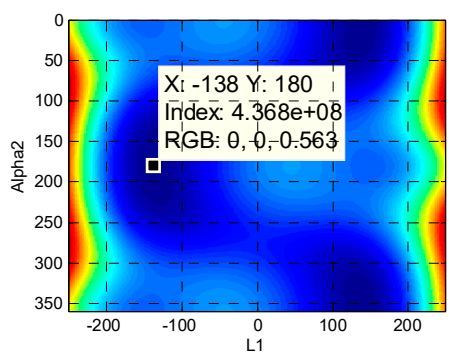

(b)

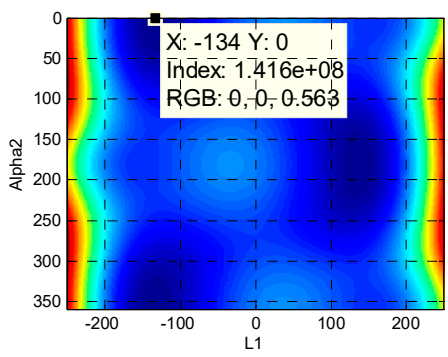

(c)

Figure A2. Part of simulation verification of numerical optimization with fixed $A_{2}$. The corresponding parameters are: (a) $A_{2}=50, i_{c}=8^{\circ} ;(\mathbf{b}) A_{2}=-200, i_{c}=12^{\circ} ;$ (c) $A_{2}=150, i_{c}=16^{\circ}$. 


\section{Appendix B}

In this Appendix, we will give a theoretical proof of the existence of no less than 2 zero points for perpendicular baseline.

Since the initial value of the argument of latitude $\alpha(t)=n_{s} t+M_{c}+\omega_{\mathcal{c}}, M_{c}+\omega_{c}$, does not affect the integral of the along-track baseline or the perpendicular baseline through the whole orbit, it may be assumed that the initial value is 0 . Thus, we have:

$$
\begin{aligned}
\widetilde{z}(t) & =\frac{-\cos (n t) \cdot\left[A_{1} \sin \left(n t+\alpha_{1}\right)+l_{1}\right]+\tan \left(i_{c} / 2\right) \cdot A_{2} \cos \left(n t+\alpha_{2}\right)}{\sqrt{\tan ^{2}\left(i_{c} / 2\right)+\cos ^{2} \alpha(t)}} \\
& =\frac{-A_{1} \cos (n t) \sin \left(n t+\alpha_{1}\right) \cdot+\left[l_{1} \cos (n t)+\tan \left(i_{c} / 2\right) \cdot A_{2} \cos \left(n t+\alpha_{2}\right)\right]}{\sqrt{\tan ^{2}\left(i_{c} / 2\right)+\cos ^{2} \alpha(t)}}
\end{aligned}
$$

According to the formula of the auxiliary angle, $l_{1} \cos \left(n t+\alpha_{0}\right)+\tan \left(i_{c} / 2\right) \cdot A_{2} \cos \left(n t+\alpha_{2}\right)$ can be transformed to $\gamma_{1}(t)=A_{3} \cos \left(n t+\alpha_{3}\right), A_{3}>0$, and $-A_{1} \cos \left(n t+\alpha_{0}\right) \sin \left(n t+\alpha_{1}\right)$ can be transformed to $\gamma_{2}(t)=A_{4}\left[\sin \left(\alpha_{4}\right)+\sin \left(2 n t+\alpha_{5}\right)\right], A_{4}>0$. Therefore the numerator of (A5) can be expressed as:

$$
\beta(t)=A_{3} \cos \left(n t+\alpha_{3}\right)+A_{4}\left[\sin \left(\alpha_{4}\right)+\sin \left(2 n t+\alpha_{5}\right)\right]
$$

We use the counter-evidence method to prove that $\beta(t)$ always has zero points. Assuming there are no zero points, let us set $\beta(t)>0$ to be true. Please note that the period of $\gamma_{1}(t)$ and $\gamma_{2}(t)$ are $T_{1}=2 \pi / n$ and $T_{2}=\pi / n$, respectively. $\gamma_{1}(t)$ is a cosine function. Therefore, there exists an interval with the length of $T_{1} / 2$ where it is not positive, that is, $\gamma_{1}(t) \leq 0$. To make $\beta(t)>0$ always hold, $\gamma_{2}(t)$ must be positive in this interval. Since $T_{2}=T_{1} / 2$, this interval is precisely the period of $\gamma_{2}(t)$. That is to say, $\gamma_{2}(t) \geq 0$ is a necessary condition. Therefore, $\sin \left(\alpha_{4}\right)=1$, thus $\gamma_{2}(t)=A_{4}\left[1+\sin \left(2 n t+\alpha_{5}\right)\right]$. It is easy to know that there is always zero points in $\gamma_{2}(t)$, which we denote as $t_{0}$. To satisfy $\beta\left(t_{0}\right)>0$, there must be $\gamma_{1}\left(t_{0}\right)>0$, which contradicts the hypothesis of the fact that $\gamma_{1}(t) \leq 0$ in the interval. This means that $\beta(t)$ has at least one zero points in this interval. Similarly, in the other half of the period $T_{1}$, the existence of zero points can be proved. Therefore, there are at least two zero points in the perpendicular baseline.

\section{References}

1. Long, T.; Hu, C.; Ding, Z.; Dong, X.; Tian, W.; Zeng, T. Geosynchronous SAR: System and Signal Processing; Springer: Berlin/Heidelberg, Germany, 2018.

2. Li, Y.; Guarnieri, A.M.; Hu, C.; Rocca, F. Performance and requirements of GEO SAR systems in the presence of Radio Frequency Interferences. Remote Sens. 2018, 10, 82. [CrossRef]

3. Hu, C.; Li, Y.; Dong, X.; Long, T. Optimal data acquisition and height retrieval in repeat-track geosynchronous SAR interferometry. Remote Sens. 2015, 7, 13367-13389. [CrossRef]

4. Hu, C.; Zhang, B.; Dong, X.; Li, Y. Geosynchronous SAR Tomography: Theory and First Experimental Verification Using Beidou IGSO Satellite. IEEE Trans. Geosci. Remote Sens. 2019, 57, 6591-6607. [CrossRef]

5. Madsen, S.N.; Edelstein, W.; DiDomenico, L.D.; LaBrecque, J. A geosynchronous synthetic aperture radar; for tectonic mapping, disaster management and measurements of vegetation and soil moisture. In Proceedings of the IGARSS 2001, Scanning the Present and Resolving the Future, International Geoscience and Remote Sensing Symposium (Cat. No.01CH37217), Sydney, NSW, Australia, 9-13 July 2001; Volume 441, pp. 447-449.

6. Guarnieri, A.M.; Leanza, A.; Recchia, A.; Tebaldini, S.; Venuti, G. Atmospheric Phase Screen in GEO-SAR: Estimation and Compensation. IEEE Trans. Geosci. Remote Sens. 2018, 56, 1668-1679. [CrossRef]

7. Hobbs, S.; Mitchell, C.; Forte, B.; Holley, R.; Snapir, B.; Whittaker, P. System Design for Geosynchronous Synthetic Aperture Radar Missions. IEEE Trans. Geosci. Remote Sens. 2014, 52, 7750-7763. [CrossRef]

8. Krieger, G.; Moreira, A.; Fiedler, H.; Hajnsek, I.; Werner, M.; Younis, M.; Zink, M. TanDEM-X: A Satellite Formation for High-Resolution SAR Interferometry. IEEE Trans. Geosci. Remote Sens. 2007, 45, 3317-3341. [CrossRef] 
9. Guarnieri, A.M.; Tebaldini, S.; Rocca, F.; Broquetas, A. GEMINI: Geosynchronous SAR for Earth Monitoring by Interferometry and Imaging. In Proceedings of the 2012 IEEE International Geoscience and Remote Sensing Symposium, Munich, Germany, 22-27 July 2012; pp. 210-213.

10. Zhu, Y.; Dong, X.; Hu, C. Formation design and baseline optimization of GEO SAR interferometry. In Proceedings of the IET International Radar Conference 2015, Hangzhou, China, 14-16 October 2015; pp. 1-5.

11. D'Errico, M. (Ed.) Distributed Space Missions for Earth System Monitoring; Springer: New York, NY, USA, 2012. [CrossRef]

12. Clohessy, W.; Wiltshire, R. Terminal guidance system for satellite rendezvous. J. Aerosp. Sci. 1960, $27,653-658$. [CrossRef]

13. Schaub, H. Spacecraft relative orbit geometry description through orbit element differences. In Proceedings of the 14th US National Congress of Theoretical and Applied Mechanics, Blacksburg, VA, USA, 23-28 June 2002.

14. Fasano, G.; Errico, M.D. Relative motion model including J/sub 2/: Derivation and application to INSAR. In Proceedings of the 2006 IEEE Aerospace Conference, Big Sky, MT, USA, 4-11 March 2006; p. 12.

15. Fasano, G.; Errico, M.D. Design of Satellite Formations for Interferometric and Bistatic SAR. In Proceedings of the 2007 IEEE Aerospace Conference, Big Sky, MT, USA, 3-10 March 2007; pp. 1-10.

16. Fasano, G.; D’Errico, M. Modeling orbital relative motion to enable formation design from application requirements. Celest. Mech. Dyn. Astron. 2009, 105, 113. [CrossRef]

17. Yicheng, J.; Yun, Z. An Optimal Baseline Design and Performance Analysis in Distributed Spaceborne SAR System. In Proceedings of the Fourth IEEE Workshop on Sensor Array and Multichannel Processing, Waltham, MA, USA, 12-14 July 2006; pp. 308-312.

18. Massonnet, D. Capabilities and limitations of the interferometric cartwheel. IEEE Trans. Geosci. Remote Sens. 2001, 39, 506-520. [CrossRef]

19. Fiedler, H.; Krieger, G.; Jochim, F.; Kirschner, M.; Moreira, A. Analysis Of Satellite Configurations For Spaceborne SAR Interferometry. In Proceedings of the Formation Flying, Toulouse, France, 29-31 October; pp. 87-96.

20. Fasano, G.; Renga, A.; D’Errico, M. Formation geometries for multistatic SAR tomography. Acta Astronaut. 2014, 96, 11-22. [CrossRef]

21. Yuan, H.; Zhou, Y.; Li, J.; Sun, M. New method of formation design for both GMTI and InSAR using distributed satellites. J. Electron. (China) 2007, 24, 821-827. [CrossRef]

22. Zhang, J.; Cao, X.; Wang, J.; Lin, X. Configuration, Orbit Design of InSAR Formation Based on Mean Elements. IEEE Trans. Aerosp. Electron. Syst. 2009, 45, 747-752. [CrossRef]

23. Xu, M.; Jia, Y.-H.; Xu, S.-J. The J 2 invariant relative configuration of spaceborne SAR interferometer for digital elevation measurement. Acta Mech. Sin. 2010, 26, 643-651. [CrossRef]

24. Moccia, A.; Fasano, G. Analysis of spaceborne tandem configurations for complementing COSMO with SAR interferometry. Eurasip J. Adv. Signal Process. 2005, 2005, 419738. [CrossRef]

25. Peterson, E.H.; Fotopoulos, G.; Zee, R.E. A feasibility assessment for low-cost InSAR formation-flying microsatellites. IEEE Trans. Geosci. Remote Sens. 2009, 47, 2847-2858. [CrossRef]

26. Hanssen, R. Radar Interferometry Data Interpretation and Error Analysis; Springer: Berlin/Heidelberg, Germany, 2001; Volume 2.

27. Hu, C.; Tian, Y.; Yang, X.; Zeng, T.; Long, T.; Dong, X. Background Ionosphere Effects on Geosynchronous SAR Focusing: Theoretical Analysis and Verification Based on the BeiDou Navigation Satellite System (BDS). IEEE J. Sel. Top. Appl. Earth Obs. Remote Sens. 2016, 9, 1143-1162. [CrossRef]

28. Rodriguez, E.; Martin, J.M. Theory and design of interferometric synthetic aperture radars. IEEE Proc. F 1992, 139, 147-159. [CrossRef]

29. Hu, C.; Li, Y.; Dong, X.; Wang, R.; Cui, C. Optimal 3D deformation measuring in inclined geosynchronous orbit SAR differential interferometry. Sci. China Inf. Sci. 2017, 60, 060303. [CrossRef]

30. Yang, J.; Fan, J.; Fan, Q. Spacecraft Orbit Dynamics and Control; China Astronautic Publishing House: Beijing, China, 1995.

31. Wie, B. Space Vehicle Dynamics and Control; American Institute of Aeronautics and Astronautics: Reston, VA, USA, 2008.

32. Hao, J. Study on Formation Configuration Control of Distributed Satellites. Ph.D. Thesis, National University of Defense Technology, Changsha, China, 2006. 
33. Eldhuset, K. A new fourth-order processing algorithm for spaceborne SAR. IEEE Trans. Aerosp. Electron. Syst. 1998, 34, 824-835. [CrossRef]

34. Cumming, I.G.; Wong, F.H. Digital processing of synthetic aperture radar data. Artech House 2005, 1.

35. Long, T.; Dong, X.; Hu, C.; Zeng, T. A New Method of Zero-Doppler Centroid Control in GEO SAR. IEEE Geosci. Remote Sens. Lett. 2011, 8, 512-516. [CrossRef]

36. Hu, C.; Li, Y.; Dong, X.; Ao, D. Avoiding the Ionospheric Scintillation Interference on Geosynchronous SAR by Orbit Optimization. IEEE Geosci. Remote Sens. Lett. 2017, 13, 1676-1680. [CrossRef]

(C) 2020 by the authors. Licensee MDPI, Basel, Switzerland. This article is an open access article distributed under the terms and conditions of the Creative Commons Attribution (CC BY) license (http://creativecommons.org/licenses/by/4.0/). 